\title{
Dilatonic black holes in higher curvature string gravity
}

\author{
P. Kanti, ${ }^{1}$ N. E. Mavromatos,${ }^{2}$ J. Rizos,${ }^{3}$ K. Tamvakis,,${ }^{3 *}$ and E. Winstanley ${ }^{2}$ \\ ${ }^{1}$ Division of Theoretical Physics, Physics Department, University of Ioannina, Ioannina GR-451 10, Greece \\ ${ }^{2}$ Department of Physics (Theoretical Physics), University of Oxford, 1 Keble Road, Oxford OX1 3NP, United Kingdom \\ ${ }^{3}$ European Organization for Nuclear Research (CERN), Theory Division, 1211 Geneva 23, Switzerland
}

(Received 10 November 1995)

\begin{abstract}
We give analytical arguments and demonstrate numerically the existence of black hole solutions of the 4D effective superstring action in the presence of Gauss-Bonnet quadratic curvature terms. The solutions possess nontrivial dilaton hair. The hair, however, is of "secondary type," in the sense that the dilaton charge is expressed in terms of the black hole mass. Our solutions are not covered by the assumptions of existing proofs of the "no-hair" theorem. We also find some alternative solutions with singular metric behavior, but finite energy. The absence of naked singularities in this system is pointed out. [S0556-2821(96)01920-0]

PACS number(s): 04.70.Bw, 04.20.Jb, 04.50.+h, 11.25.Mj
\end{abstract}

\section{INTRODUCTION}

It has become evident in recent years that the properties of black holes are modified when the theory of matter fields has sufficient structure. In the presence of the low-energy degrees of freedom characteristic of string theory [1], i.e., dilatons, axions, and Abelian or Yang-Mills fields, it is possible to have nontrivial static configurations for these fields outside the horizon, i.e., to have black holes with hair [2,3]. It is not clear, however, whether these cases, in which the "nohair theorem" [4] does not apply [5], represent stable solutions. Explicit black hole solutions have been found also in string-effective theories involving higher-order curvature corrections to the Einstein gravity. They exhibit secondary hair of the dilaton, axion, and modulus fields. The solutions were approximate, in the sense that only a perturbative analysis to $O\left(\alpha^{\prime}\right)$ [6] and $O\left(\alpha^{\prime 2}\right)$ [7] has been performed. This analysis motivates the search for exact (to all orders in $\alpha^{\prime}$ ) solutions within the framework of curvature-squared corrections to Einstein's theory. Although the effect of the higher-order curvature terms is not small for energy scales of order $\alpha^{\prime}$, from a local field theory point of view it makes sense to look for this kind of solutions, with the hope of drawing some useful conclusions that might be of relevance to the low-energy limit of string theories.

In the present article we shall demonstrate the existence of black hole solutions of the Einstein-dilaton system in the presence of the higher-derivative, curvature squared terms. These solutions will be endowed with a nontrivial dilaton field outside the horizon, thus possessing dilaton hair. The treatment of the quadratic terms will be nonperturbative and the solutions are present for any value of $\alpha^{\prime} / g^{2}$. What we shall argue in this paper is that the presence of these terms provides the necessary "repulsion" in the effective theory that balances the gravitational attraction, thereby leading to black holes dressed with nontrivial classical dilaton hair. An analogous phenomenon occurs already in the case of Einstein-Yang-Mills systems [3]. There the presence of the

\footnotetext{
*On leave of absence from Physics Department, University of Ioannina, Ioannina, Greece.
}

non-Abelian gauge field repulsion balances the gravitational attraction leading to black hole solutions with nontrivial gauge and scalar (in Higgs systems) hair.

It is useful to discuss briefly the situation in effective theories obtained from the string. We shall concentrate on the bosonic part of the gravitational multiplet which consists of the dilaton, graviton, and antisymmetric tensor fields. In this work we shall ignore the antisymmetric tensor for simplicity. ${ }^{1}$ As is well known in low-energy effective field theory, there are ambiguities in the coefficients of such terms, due to the possibility of local field redefinitions which leave the $S$-matrix amplitudes of the effective field theory invariant, according to the equivalence theorem. To $O\left(\alpha^{\prime}\right)$ the freedom of such redefinitions is restricted to two generic structures, which cannot be removed by further redefinitions [8]. One is a curvature-squared combination, and the other is a four-derivative dilaton term. Thus, a generic form of the string-inspired $O\left(\alpha^{\prime}\right)$ corrections to Einstein's gravitation have the form

$$
\mathcal{L}=-\frac{1}{2} R-\frac{1}{4}\left(\partial_{\mu} \phi\right)^{2}+\frac{\alpha^{\prime}}{8 g^{2}} e^{\phi}\left[c_{1} \mathcal{R}^{2}+c_{2}\left(\partial_{\rho} \phi\right)^{4}\right]
$$

where $\alpha^{\prime}$ is the Regge slope, $g^{2}$ is some gauge coupling constant (in the case of the heterotic string that we concentrate for physical reasons), and $\mathcal{R}^{2}$ is a generic curvaturedependent quadratic structure, which can always be fixed to correspond to the Gauss-Bonnet (GB) invariant

$$
R_{\mathrm{GB}}^{2}=R_{\mu \nu \rho \sigma} R^{\mu \nu \rho \sigma}-4 R_{\mu \nu} R^{\mu \nu}+R^{2} .
$$

The coefficients $c_{1}, c_{2}$ are fixed by comparison with stringscattering amplitude computations, or $\sigma$-model $\beta$-function analysis. It is known that in the three types of string theories, bosonic, closed-type II superstring, and heterotic strings, the

\footnotetext{
${ }^{1}$ In four dimensions, the antisymmetric tensor field leads to the axion hair, already discussed in Ref. [6]. Modulo unexpected surprises, we do not envisage problems associated with its presence as regards the results discussed in this work, and, hence, we ignore it for simplicity.
} 
ratio of the $c_{1}$ coefficients is 2:0:1, respectively [8]. The case of superstring II effective theory, then, is characterized by the absence of curvature-squared terms. In such theories the fourth-order dilaton terms can still be, and in fact they are, present. In such a case, it is straightforward to see from the modern proof of the no-scalar-hair theorem of Ref. [4] that such theories cannot sustain to order $O\left(\alpha^{\prime}\right)$, any nontrivial dilaton hair. On the other hand, the presence of curvaturesquared terms can drastically change the situation, as we shall discuss in this article. There is a simple reason to expect that in this case the no-scalar-hair theorem can be bypassed. In the presence of curvature-squared terms, the modified Einstein's equation leads to an effective stress tensor that involves the gravitational field. This implies that the assumption of positive definiteness of the time component of this tensor, which in the Einstein case is the local energy density of the field, may, and as we shall show it does indeed, break down. The second, but equally important, reason is that as a result of the higher-curvature terms, there is an induced modification of the relation $T_{t}^{t}=T_{\theta}^{\theta}$ between the time and angular components of the stress tensor, which was valid in the case of spherically symmetric Einstein theories of Ref. [4].

The structure of the article is the following: In Sec. II we give analytic arguments for the existence of scalar (dilaton) hair of the black hole solution, which bypasses the conditions for the no-hair theorem. In Sec. III we present an analysis of the black hole solutions. In Sec. IV we discuss alternative solutions, some of which are interesting due to the finite energy-momentum tensor they possess. Finally, conclusions and outlook are presented in Sec. V.

\section{EXISTENCE OF HAIR IN GRAVITY WITH A GAUSS- BONNET TERM: ANALYTIC ARGUMENTS}

Following the above discussion we shall ignore, for simplicity, the fourth-derivative dilaton terms in Eq. (1), setting from now on $c_{2}=0$. However, we must always bear in mind that such terms are nonzero in realistic effective string cases, once the GB combination is fixed for the gravitational $O\left(\alpha^{\prime}\right)$ parts. Then, the Lagrangian for dilaton gravity with a Gauss-Bonnet term reads

$$
\mathcal{L}=-\frac{1}{2} R-\frac{1}{4}\left(\partial_{\mu} \phi\right)^{2}+\frac{\alpha^{\prime}}{8 g^{2}} e^{\phi} R_{\mathrm{GB}}^{2}
$$

where $R_{\mathrm{GB}}^{2}$ is the Gauss-Bonnet (GB) term (2).

As we mentioned in the Introduction, although we view Eq. (3) as a heterotic-string effective action, for simplicity, in this paper we shall ignore the modulus and axion fields, assuming reality of the dilaton $\left(S=e^{\phi}\right.$ in the notation of Ref. [6]). We commence our analysis by noting that the dilaton field and Einstein's equations derived from Eq. (3) are

$$
\begin{gathered}
\frac{1}{\sqrt{-g}} \partial_{\mu}\left[\sqrt{-g} \partial^{\mu} \phi\right]=-\frac{\alpha^{\prime}}{4 g^{2}} e^{\phi} R_{\mathrm{GB}}^{2} \\
R_{\mu \nu}-\frac{1}{2} g_{\mu \nu} R=-\frac{1}{2} \partial_{\mu} \phi \partial_{\nu} \phi+\frac{1}{4} g_{\mu \nu}\left(\partial_{\rho} \phi\right)^{2}-\alpha^{\prime} \mathcal{K}_{\mu \nu},
\end{gathered}
$$

where

$$
\mathcal{K}_{\mu \nu}=\left(g_{\mu \rho} g_{\nu \lambda}+g_{\mu \lambda} g_{\nu \rho}\right) \eta^{\kappa \lambda \alpha \beta} D_{\gamma}\left[\widetilde{R}^{\rho \gamma}{ }_{\alpha \beta} \partial_{\kappa} f\right],
$$

and

$$
\begin{gathered}
\eta^{\mu \nu \rho \sigma}=\epsilon^{\mu \nu \rho \sigma}(-g)^{-1 / 2}, \\
\epsilon^{0 i j k}=-\epsilon_{i j k}, \\
\widetilde{R}_{\kappa \lambda}^{\mu \nu}=\eta^{\mu \nu \rho \sigma} R_{\rho \sigma \kappa \lambda}, \\
f=\frac{e^{\phi}}{8 g^{2}} .
\end{gathered}
$$

From the right-hand side (RHS) of the modified Einstein's equation (5), one can construct a conserved "energymomentum tensor,' $D_{\mu} T^{\mu \nu}=0$,

$$
T_{\mu \nu}=\frac{1}{2} \partial_{\mu} \phi \partial_{\nu} \phi-\frac{1}{4} g_{\mu \nu}\left(\partial_{\rho} \phi\right)^{2}+\alpha^{\prime} \mathcal{K}_{\mu \nu}
$$

It should be stressed that the time component of $-T_{\mu \nu}$, which in Einstein's gravity would correspond to the local energy density $\mathcal{E}$, may not be positive. Indeed, as we shall see later on, for spherically symmetric space-times, there are regions where this quantity is negative. The reason is that, as a result of the higher-derivative GB terms, there are contributions of the gravitational field itself to $T_{\mu \nu}$. From a string theory point of view, this is reflected in the fact that the dilaton is part of the string gravitational multiplet. Thus, this is the first important indication on the possibility of evading the no-scalar-hair theorem of Ref. [4] in this case. However, this by itself is not sufficient for a rigorous proof of an evasion of the no-hair conjecture. We shall come to this point later on.

At the moment, let us consider a spherically symmetric space-time having the metric

$$
d s^{2}=-e^{\Gamma} d t^{2}+e^{\Lambda} d r^{2}+r^{2}\left(d \theta^{2}+\sin ^{2} \theta d \varphi^{2}\right),
$$

where $\Gamma, \Lambda$ depend on $r$ solely. Using the above ansatz, the dilaton equation as well as the $(t t),(r r)$, and $(\theta \theta)$ component of the Einstein's equations take the form

$$
\begin{aligned}
& \phi^{\prime \prime}+\phi^{\prime}\left(\frac{\Gamma^{\prime}-\Lambda^{\prime}}{2}+\frac{2}{r}\right)=\frac{\alpha^{\prime} e^{\phi}}{g^{2} r^{2}}\left(\Gamma^{\prime} \Lambda^{\prime} e^{-\Lambda}+\left(1-e^{-\Lambda}\right)\right. \\
& \left.\times\left[\Gamma^{\prime \prime}+\frac{\Gamma^{\prime}}{2}\left(\Gamma^{\prime}-\Lambda^{\prime}\right)\right]\right), \\
& \Lambda^{\prime}\left(1+\frac{\alpha^{\prime} e^{\phi}}{2 g^{2} r} \phi^{\prime}\left(1-3 e^{-\Lambda}\right)\right)=\frac{r \phi^{\prime 2}}{4}+\frac{1-e^{\Lambda}}{r}+\frac{\alpha^{\prime} e^{\phi}}{g^{2} r}\left(\phi^{\prime \prime}\right. \\
& \left.+\phi^{\prime 2}\right)\left(1-e^{-\Lambda}\right) \text {, } \\
& \Gamma^{\prime}\left(1+\frac{\alpha^{\prime} e^{\phi}}{2 g^{2} r} \phi^{\prime}\left(1-3 e^{-\Lambda}\right)\right)=\frac{r \phi^{\prime 2}}{4}+\frac{e^{\Lambda}-1}{r},
\end{aligned}
$$




$$
\begin{aligned}
\Gamma^{\prime \prime}+\frac{\Gamma^{\prime}}{2}\left(\Gamma^{\prime}-\Lambda^{\prime}\right)+\frac{\Gamma^{\prime}-\Lambda^{\prime}}{r}= & -\frac{\phi^{\prime 2}}{2}+\frac{\alpha^{\prime} e^{\phi-\Lambda}}{g^{2} r} \\
& \times\left(\phi^{\prime} \Gamma^{\prime \prime}+\left(\phi^{\prime \prime}+\phi^{\prime 2}\right) \Gamma^{\prime}\right. \\
& \left.+\frac{\phi^{\prime} \Gamma^{\prime}}{2}\left(\Gamma^{\prime}-3 \Lambda^{\prime}\right)\right)
\end{aligned}
$$

Before we proceed to study the above system it is useful to note that if we turn off the Gauss-Bonnet term, Eq. (10) can be integrated to give $\phi^{\prime} \sim\left(1 / r^{2}\right) e^{(\Lambda-\Gamma) / 2}$. A black hole solution should have at the horizon $r_{h}$ the behavior $e^{-\Gamma}$, $e^{\Lambda} \rightarrow \infty$. Therefore, the radial derivative of the dilaton would diverge on the horizon resulting into a divergent energymomentum tensor

$$
T_{t}^{t}=-T_{r}^{r}=T_{\theta}^{\theta}=-\frac{e^{-\Lambda}}{4} \phi^{\prime 2} \rightarrow \infty .
$$

Rejecting this solution, we are left with the standard Schwarzschild solution and a trivial ( $\phi=$ const) dilaton, in agreement with the no-hair theorem. This behavior will be drastically modified by the Gauss-Bonnet term.

The $r$ component of the energy-momentum conservation equations reads

$$
\left(e^{\Gamma / 2} r^{2} T_{r}^{r}\right)^{\prime}=\frac{1}{2} e^{\Gamma / 2} r^{2}\left[\Gamma^{\prime} T_{t}^{t}+\frac{4}{r} T_{\theta}^{\theta}\right],
$$

where the prime denotes differentiation with respect to $r$. The spherical symmetry of the space-time implies $T_{\theta}^{\theta}=T_{\varphi}^{\varphi}$. Integrating over the radial coordinate $r$ from the horizon $r_{h}$ to generic $r$ yields

$$
T_{r}^{r}(r)=\frac{e^{-\Gamma / 2}}{2 r^{2}} \int_{r_{h}}^{r} e^{\Gamma / 2} r^{2}\left[\Gamma^{\prime} T_{t}^{t}+\frac{4}{r} T_{\theta}^{\theta}\right] d r .
$$

The boundary terms on the horizon vanish, since scalar invariants such as $T_{\alpha \beta} T^{\alpha \beta}$ are finite there. For the first derivative of $T_{r}^{r}$, we have

$$
\left(T_{r}^{r}\right)^{\prime}(r)=\frac{e^{-\Gamma / 2}}{r^{2}}\left(e^{\Gamma / 2} r^{2}\right)^{\prime}\left(T_{t}^{t}-T_{r}^{r}\right)+\frac{2}{r}\left(T_{\theta}^{\theta}-T_{r}^{r}\right) .
$$

Taking into account Eqs. (8) and (9), one easily obtains

$$
\begin{aligned}
T_{t}^{t}= & -e^{-\Lambda} \frac{\phi^{\prime 2}}{4}-\frac{\alpha^{\prime}}{g^{2} r^{2}} e^{\phi-\Lambda}\left(\phi^{\prime \prime}+\phi^{\prime 2}\right)\left(1-e^{-\Lambda}\right) \\
& +\frac{\alpha^{\prime}}{2 g^{2} r^{2}} e^{\phi-\Lambda} \phi^{\prime} \Lambda^{\prime}\left(1-3 e^{-\Lambda}\right), \\
T_{r}^{r}= & e^{-\Lambda} \frac{\phi^{\prime 2}}{4}-\frac{\alpha^{\prime}}{2 g^{2} r^{2}} e^{\phi-\Lambda} \phi^{\prime} \Gamma^{\prime}\left(1-3 e^{-\Lambda}\right), \\
T_{\theta}^{\theta}= & -e^{-\Lambda} \frac{\phi^{\prime 2}}{4}+\frac{\alpha^{\prime}}{2 g^{2} r} e^{\phi-2 \Lambda}\left[\Gamma^{\prime \prime} \phi^{\prime}+\Gamma^{\prime}\left(\phi^{\prime \prime}+\phi^{\prime 2}\right)\right. \\
& \left.+\frac{\Gamma^{\prime} \phi^{\prime}}{2}\left(\Gamma^{\prime}-3 \Lambda^{\prime}\right)\right] .
\end{aligned}
$$

In the relations (18) there lies the second reason for a possibility of an evasion of the no-hair conjecture. Due to the presence of the higher-curvature contributions, the relation $T_{t}^{t}=T_{\theta}^{\theta}$ assumed in Ref. [4] is no longer valid. The alert reader must have noticed, then, the similarity of the role played by the Gauss-Bonnet $O\left(\alpha^{\prime}\right)$ terms in the Lagrangian (3) with the case of the non-Abelian gauge black holes studied in Ref. [5]. There the presence of the non-Abelian gauge field repulsive forces also lead to nontrivial contributions to $T_{\theta}^{\theta} \neq T_{t}^{t}$ leading to a sort of "balancing"' between this repulsion and the gravitational attraction. We stress, once again, however, that in our case both the nonpositivity of the "energy-density", $T_{t}^{t}$ and the modified relation $T_{t}^{t} \neq T_{\theta}^{\theta}$ play equally important roles in leading to a possibility of having nontrivial classical scalar (dilaton) hair in GB black holes' systems. Below, we shall demonstrate rigorously this, by showing that there is no contradiction between the results following from the conservation equation of the "energymomentum tensor" $T_{\mu \nu}$ and the field equations, in the presence of nontrivial dilaton hair.

Far away from the origin, the unknown functions $\phi(r)$, $e^{\Lambda(r)}$, and $e^{\Gamma(r)}$ can be expanded in a power series in $1 / r$. These expansions, substituted back into the equations, are finally expressed in terms of three parameters only, chosen to be $\phi_{\infty}$, the asymptotic value of the dilaton, the ArnowittDeser-Misner (ADM) mass $M$, and the dilaton charge $D$ defined as [10]

$$
D=-\frac{1}{4 \pi} \int d^{2} \Sigma^{\mu} D_{\mu} \phi
$$

where the integral is over a two sphere at spatial infinity. The asymptotic solutions are

$$
\begin{gathered}
e^{\Lambda(r)}=1+\frac{2 M}{r}+\frac{16 M^{2}-D^{2}}{4 r^{2}}+O\left(1 / r^{3}\right), \\
e^{\Gamma(r)}=1-\frac{2 M}{r}+O\left(1 / r^{3}\right) \\
\phi(r)=\phi_{\infty}+\frac{D}{r}+\frac{M D}{r^{2}}+\mathcal{O}\left(1 / r^{3}\right) .
\end{gathered}
$$

To check the possibility of the evasion of the no-hair conjecture we first consider the asymptotic behavior of $T_{r}^{r}$ as $r \rightarrow \infty$. Since $\Gamma^{\prime}$ and $\Lambda^{\prime}=O\left(1 / r^{2}\right)$ as $r \rightarrow \infty$, we have the asymptotic behavior

$$
\begin{gathered}
T_{r}^{r} \sim \frac{1}{4}\left(\phi^{\prime}\right)^{2}+O\left(\frac{1}{r^{6}}\right), \\
T_{\theta}^{\theta} \sim-\frac{1}{4}\left(\phi^{\prime}\right)^{2}+O\left(\frac{1}{r^{6}}\right) .
\end{gathered}
$$

In this limit, $e^{\Gamma / 2} \rightarrow 1$, and so the leading behavior of $\left(T_{r}^{r}\right)^{\prime}$ is

$$
\left(T_{r}^{r}\right)^{\prime} \sim \frac{2}{r}\left(T_{\theta}^{\theta}-T_{r}^{r}\right) \sim-\frac{1}{r}\left(\phi^{\prime}\right)^{2}<0 \text { as } r \rightarrow \infty .
$$

Thus, $T_{r}^{r}$ is positive and decreasing as $r \rightarrow \infty$. 
We now turn to the behavior of the unknown functions at the event horizon. When $r \sim r_{h}$, we make the ansatz

$$
\begin{gathered}
e^{-\Lambda(r)}=\lambda_{1}\left(r-r_{h}\right)+\lambda_{2}\left(r-r_{h}\right)^{2}+\cdots, \\
e^{\Gamma(r)}=\gamma_{1}\left(r-r_{h}\right)+\gamma_{2}\left(r-r_{h}\right)^{2}+\cdots, \\
\phi(r)=\phi_{h}+\phi_{h}^{\prime}\left(r-r_{h}\right)+\phi_{h}^{\prime \prime}\left(r-r_{h}\right)^{2}+\cdots,
\end{gathered}
$$

with the subscript $h$ denoting the value of the respective quantities at the horizon. We shall demonstrate in the Appendix that this is the most general asymptotic solution with $\Gamma^{\prime} \rightarrow \infty \phi, \phi^{\prime}, \phi^{\prime \prime}$ finite. As we can see, $\phi\left(r_{h}\right) \sim$ const while $\Gamma^{\prime}$ and $\Lambda^{\prime}$ diverge as $\left(r-r_{h}\right)^{-1}$ and $-\left(r-r_{h}\right)^{-1}$, respectively. Then, the behavior of the components of the energymomentum tensor near the horizon is

$$
\begin{gathered}
T_{r}^{r}=-\frac{\alpha^{\prime}}{2 g^{2} r^{2}} e^{\phi-\Lambda} \phi^{\prime} \Gamma^{\prime}+O\left(r-r_{h}\right), \\
T_{t}^{t}=\frac{\alpha^{\prime}}{2 g^{2} r^{2}} e^{\phi-\Lambda} \phi^{\prime} \Lambda^{\prime}+O\left(r-r_{h}\right), \\
T_{\theta}^{\theta}=\frac{\alpha^{\prime}}{2 g^{2} r} e^{\phi-2 \Lambda}\left[\Gamma^{\prime \prime} \phi^{\prime}+\frac{\Gamma^{\prime} \phi^{\prime}}{2}\left(\Gamma^{\prime}-3 \Lambda^{\prime}\right)\right]+O\left(r-r_{h}\right) .
\end{gathered}
$$

Taking into account the above expressions, the leading behavior of $T_{r}^{r}$ near the horizon is

$$
T_{r}^{r}(r) \sim-\frac{e^{-\Gamma / 2}}{r^{2}} \int_{r_{h}}^{r} \frac{\alpha^{\prime}}{4 g^{2}} e^{\Gamma / 2}\left(\Gamma^{\prime}\right)^{2} e^{-\Lambda} e^{\phi} \phi^{\prime} d r+O\left(r-r_{h}\right) .
$$

Therefore, one observes that for $r$ sufficiently close to the event horizon, $T_{r}^{r}$ has opposite sign to $\phi^{\prime}$.

For $\left(T_{r}^{r}\right)^{\prime}$ near the horizon, we have

$$
\begin{aligned}
\left(T_{r}^{r}\right)^{\prime}(r)= & \frac{\alpha^{\prime}}{2 g^{2}} \frac{e^{\phi}}{r^{2}} e^{-\Lambda}\left\{-\Gamma^{\prime}\left(\phi^{\prime \prime}+\phi^{\prime 2}\right)+\phi^{\prime}\left[\frac{\Gamma^{\prime}}{2}\left(\Gamma^{\prime}+\Lambda^{\prime}\right)\right.\right. \\
& \left.\left.+2 e^{-\Lambda} \Gamma^{\prime \prime}-\frac{2}{r} \Lambda^{\prime}\right]\right\}-\frac{1}{4} \Gamma^{\prime} e^{-\Lambda} \phi^{\prime 2}+O\left(r-r_{h}\right),
\end{aligned}
$$

where $\Gamma^{\prime}+\Lambda^{\prime} \sim 1$ for $r \sim r_{h}$. Adding the $(t t)$ and $(r r)$ components of the Einstein's equations, we obtain at the event horizon

$$
\Gamma^{\prime}+\Lambda^{\prime}=\frac{1}{\mathcal{F}}\left[\frac{1}{2} r_{h}{\phi_{h}^{\prime}}^{2}+\frac{\alpha^{\prime}}{g^{2}} \frac{e^{\phi_{h}}}{r_{h}}\left(\phi_{h}{ }^{\prime}+{\phi_{h}^{\prime}}^{2}\right)\right]+\mathcal{O}\left(r-r_{h}\right),
$$

where

$$
\mathcal{F}=1+\frac{\alpha^{\prime}}{2 g^{2}} \frac{e^{\phi_{h}}}{r_{h}} \phi_{h}^{\prime}
$$

From the $(\theta \theta)$ component, we obtain

$$
\begin{aligned}
e^{-2 \Lambda} \Gamma^{\prime \prime} & =-\frac{1}{2} e^{-2 \Lambda}\left(\Gamma^{\prime}\right)^{2}+\frac{1}{2} e^{-2 \Lambda} \Gamma^{\prime} \Lambda^{\prime}+O\left(r-r_{h}\right) \\
& =-\frac{1}{r_{h}^{2} \mathcal{F}^{2}}+O\left(r-r_{h}\right) .
\end{aligned}
$$

Substituting all the above formulas into Eq. (28) yields, near $r_{h}$

$$
\begin{aligned}
\left(T_{r}^{r}\right)^{\prime}(r) \sim & -\frac{1}{4} \frac{\phi_{h}^{\prime 2}}{r_{h}^{2} \mathcal{F}}-\frac{\alpha^{\prime}}{2 g^{2}} \frac{e^{\phi_{h}}}{r_{h}^{3} \mathcal{F}^{2}}\left(\phi_{h}^{\prime \prime}+\phi_{h}^{\prime 2}\right) \\
& -\frac{\alpha^{\prime}}{4 g^{4}} \frac{e^{2} \phi_{h}}{r_{h}^{5} \mathcal{F}^{2}} \phi_{h}^{\prime 2}+O\left(r-r_{h}\right) .
\end{aligned}
$$

Next, we turn to the dilaton equation (10). At $r \sim r_{h}$, it takes the form

$$
\frac{\phi_{h}^{\prime}}{r_{h} \mathcal{F}}=-\frac{3}{\mathcal{F}^{2}} \frac{\alpha^{\prime}}{g^{2}} \frac{e^{\phi_{h}}}{r_{h}^{4}}+O\left(r-r_{h}\right)
$$

Substituting for $\mathcal{F}$ [Eq. (30)], the following equation for $\phi_{h}^{\prime}$ is derived:

$$
\frac{\alpha^{\prime}}{2 g^{2}} \frac{e^{\phi_{h}}}{r_{h}} \phi_{h}^{\prime 2}+\phi_{h}^{\prime}+\frac{3}{r_{h}^{3}} \frac{\alpha^{\prime}}{g^{2}} e^{\phi_{h}}=0
$$

which has as solutions

$$
\phi_{h}^{\prime}=\frac{g^{2}}{\alpha^{\prime}} r_{h} e^{-\phi_{h}}\left(-1 \pm \sqrt{1-\frac{6\left(\alpha^{\prime}\right)^{2}}{g^{4}} \frac{e^{2 \phi_{h}}}{r_{h}^{4}}}\right) .
$$

As we will see below the relation (35) guarantees the finiteness of $\phi_{h}^{\prime \prime}$, and hence of the "local density", $T_{t}^{t}$ [Eq. (18)]. Both these solutions for $\phi_{h}^{\prime}$ are negative, and hence, since $T_{r}^{r}\left(r_{h}\right)$ has the opposite sign to $\phi_{h}^{\prime}, T_{r}^{r}$ will be positive sufficiently close to the horizon. Since $T_{r}^{r} \geqslant 0$ also at infinity, we observe that there is no contradiction with Einstein's equations, thereby allowing for the existence of black holes with scalar hair. We observe that near the horizon the quantity $\mathcal{E}\left(-T_{t}^{t}\right)$, which in Einstein's gravitation would be the local energy density of the field $\phi$, is negative. As we mentioned earlier, this constitutes one of the reasons one should expect an evasion of the no-scalar-hair conjecture in this black hole space-time. Crucial also for this result was the presence of additional terms in Eq. (18), leading to $T_{t}^{t}$ $\neq T_{\theta}^{\theta}$. Both of these features, whose absence in the case of Einstein-scalar gravity was crucial for the modern proof of the no-hair theorem, owe their existence in the presence of the higher-order $O\left(\alpha^{\prime}\right)$ corrections in Eq. (3).

The physical importance of the restriction (35) lies in the fact that according to this relation, black hole solutions of a given horizon radius can only exist if the coupling constant of the Gauss-Bonnet term in Eq. (3) is smaller than a critical value, set by the magnitude of the horizon scale. In fact from Eq. (35), reality of $\phi_{h}^{\prime}$ is guaranteed if and only if

$$
e^{\phi_{h}}<\frac{g^{2}}{\sqrt{6} \alpha^{\prime}} r_{h}^{2}
$$


TABLE I. Corresponding values for $\phi_{h}$ and $\phi_{\infty}$.

\begin{tabular}{lcccc}
\hline \hline$\phi_{h}$ & $\phi_{\infty}$ & $r_{h}$ & $2 M$ & $D$ \\
\hline-0.900 & -1.59656 & 2.22172 & 2.40514 & 0.686968 \\
-1.00 & -1.62901 & 2.25806 & 2.41212 & 0.655905 \\
-1.50 & -1.88561 & 2.56717 & 2.63528 & 0.500778 \\
-2.00 & -2.23788 & 3.06161 & 3.09293 & 0.382030 \\
-3.00 & -3.08963 & 4.68710 & 4.69399 & 0.226401 \\
-5.00 & -5.01232 & 12.2578 & 12.2581 & 0.082250 \\
-10.0 & -10.0001 & 148.421 & 148.422 & 0.005937 \\
\hline \hline
\end{tabular}

In this picture, $\beta \equiv \frac{1}{4} e^{\phi_{h}}$ can then be viewed as the (appropriately normalized with respect to the Einstein term) coupling constant of the GB term in the effective Lagrangian (3). For a black hole of unit horizon radius $r_{h}=1$, the critical value of $\beta$, above which black hole solutions cannot exist, is then $\beta_{c}=g^{2} / 4 \sqrt{6} \alpha^{\prime}$. One is tempted to compare the situation with the case of $\mathrm{SU}(2)$ sphaleron solutions in the presence of Gauss-Bonnet terms [9]. Numerical analysis of sphaleron solutions in such systems reveals the existence of a critical value for the GB coefficient above which solutions do not exist. In the sphaleron case this number depends on the number of nodes of the Yang-Mills gauge field. In our case, if one fixes the position of the horizon, then it seems that in order to construct black hole solutions with this horizon size the GB coefficient has to satisfy Eq. (36). Thus, a way of interpreting Eq. (35) is to view it as providing a necessary condition for the absence of naked singularities in spacetime. To understand better this latter point, we should consider the scalar curvature in the limit $r \rightarrow r_{h}$. It is

$$
R=\frac{2}{r^{2}}\left(1-\lambda_{1} r\right)^{2}+O\left(r-r_{h}\right)
$$

The constant $\lambda_{1}$ defined in Eq. (25) can be easily deduced from Eq. (12) in the limit $r \rightarrow r_{h}$ to be $\lambda_{1}=2 /\left(e^{\phi_{h}} \phi_{h}^{\prime}+2 r_{h}\right)$. Substituting $\lambda_{1}$, we obtain

$$
R=\frac{2}{r_{h}^{2}}\left(\frac{1 \mp \sqrt{1-\frac{6\left(\alpha^{\prime}\right)^{2}}{g^{4}} \frac{e^{2 \phi_{h}}}{r_{h}^{4}}}}{1 \pm \sqrt{1-\frac{6\left(\alpha^{\prime}\right)^{2}}{g^{4}} \frac{e^{2 \phi_{h}}}{r_{h}^{4}}}}\right)^{2}
$$

This expression shows that the curvature is singular at $r_{h} \rightarrow 0$, i.e., when the horizon shrinks. The point $r_{h}=0$ can be reached only when $\phi_{h}=-\infty$. Thus, the inequality (36), in a sense, forbids $r_{h}$ to become zero and reveal the singularity.

Above, we have argued on the possibility of having black holes in the system (3) that admit nontrivial dilaton hair outside their horizon. The key is the bypassing of the no-hair theorem [4], as a result of the curvature-squared terms. In what follows we shall write down explicit solutions of the equations of motion originating from Eq. (3) and provide evidence for the existence of black hole solutions to all orders in $\alpha^{\prime}$. Unfortunately, a complete analytic treatment of these equations is not feasible, and one has to use numerical methods. This complicates certain things, in particular, it does not allow for a clear view of what happens inside the horizon, thereby not giving any information on the curvature singularity structure.

\section{NUMERICAL ANALYSIS}

We now proceed to the numerical integration. Starting from the solution (A8)-(A10), at $r=r_{h}+\epsilon, \epsilon \simeq 10^{-8}$, we integrate the system (A3), (A4) towards $r \rightarrow \infty$ using the fourth-order Runge-Kutta method with an automatic step procedure and accuracy $10^{-8}$. The integration stops when the flat space-time asymptotic limit (20)-(22) is reached. Note that only the choice $\sigma=+1$ in Eq. (A8) leads to solutions which have the desired behavior (22) for the dilaton field at infinity. Since $\Lambda(r)$ is not an independent variable, and $\phi_{h}^{\prime}$ is related to $\phi_{h}$ and $r_{h}$ through Eq. (A8), it seems that the only independent parameters of the problem are $\phi_{h}$, $r_{h}$, and $\gamma_{1}$. Note that the equations of motion do not yield any constraint for $\gamma_{1}$. This is due to the fact that the equations of motion (10)-(13) do not involve $\Gamma(r)$ but only $\Gamma^{\prime}(r)$. Thus, only $\Gamma^{\prime}(r)$ can be determined by them and in order to obtain $\Gamma(r)$ a final integration has to be performed. This integration involves an integration constant $\gamma_{1}$ which will be fixed by demanding the asymptotically flat limit (21). Thus, the only independent parameters are just $\phi_{h}$ and $r_{h}$.

Note, however, that the equations of motion remain invariant under a shift $\phi \rightarrow \phi+\phi_{0}$ as long as it is accompanied by a radial rescaling $r \rightarrow r e^{\phi_{0} / 2}$. Due to the above invariance it is sufficient to vary only one of $r_{h}$ and $\phi_{h}$. For calculational convenience we set $r_{h}=1$. It turns out that for every value of the shooting parameter $\phi_{h}$, satisfying the inequality (36), there is a black hole solution regular at infinity. This is another new feature which follows from the presence of the Gauss-Bonnet term. It is interesting to note that beyond a value of the input parameter $\phi_{h}$, the black hole reaches quickly its Schwarzschild value $2 M=r_{h}$. Note that the dilaton charge does not vanish. The approach to the Schwarzschild limit is a reflection of the fact that as we move to $\phi_{h}$ values smaller than the critical value $\ln (1 / \sqrt{6})$ $=-0.89588 \ldots$, the variation of the dilaton field $\phi(r)$ with $r$ becomes eventually weaker and the dilaton ultimately behaves as a constant. For an almost constant dilaton the Gauss-Bonnet term is irrelevant and the mass takes up its Schwarzschild value. At the end of our numerical calculation and in order to fix a unique scale, we impose on the dilaton field the asymptotic condition $\phi_{\infty}=0$. This requires a shift $\phi \rightarrow \phi-\phi_{\infty}$ as well as a rescaling $r \rightarrow r e^{-\phi_{\infty} / 2}$. Since the radial coordinate has been rescaled, the other two asymptotic 


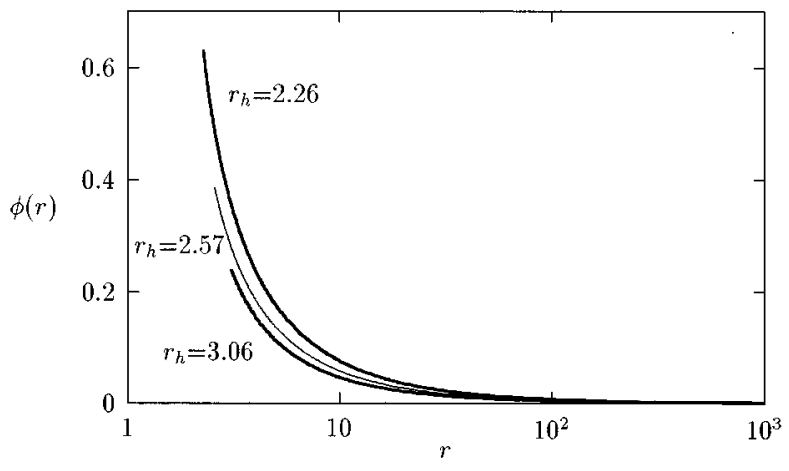

FIG. 1. Dilaton field for black hole solutions. Each curve corresponds to a different solution characterized by a different initial value of $\phi_{h}$.

parameters, $M$ and $D$, are also rescaled according to the rule: $M \rightarrow M e^{-\phi_{\infty} / 2}$ and $D \rightarrow D e^{-\phi_{\infty} / 2}$. In Table I we display corresponding values for $\phi_{h}$ and $\phi_{\infty}$ as well as the rescaled values of $r_{h}, 2 M$, and $D$. Plots involving the dilaton field $\phi(r)$, for three different allowed values of the solution parameter $\phi_{h}$, are given in Fig. 1. The metric functions $e^{\Lambda(r)}, e^{\Gamma(r)}$, as well as the three components $T_{t}^{t} T_{r}^{r}$, and $T_{\theta}^{\theta}$ of the energy-momentum tensor for $r_{h}=1$, are presented in Figs. 2 and 3, respectively.

As we said before, the solution near the horizon is characterized by the parameter $\phi_{h}$. However, the parameters that characterize the solution near infinity (20)-(22) are $M$ and $D$. From this, we can infer that a relation must hold between the above parameters in order to be able to classify our solution as a one-parameter family of black hole solutions. After some manipulation, the set of equations (10)-(13) can be rearranged to yield the identity

$$
\begin{aligned}
& \frac{d}{d r}\left(r^{2} e^{(\Gamma-\Lambda) / 2}\left(\Gamma^{\prime}-\phi^{\prime}\right)-\frac{\alpha^{\prime} e^{\phi}}{g^{2}} e^{(\Gamma-\Lambda) / 2}\left[\left(1-e^{-\Lambda}\right)\right.\right. \\
& \left.\left.\times\left(\phi^{\prime}-\Gamma^{\prime}\right)+e^{-\Lambda} r \phi^{\prime} \Gamma^{\prime}\right]\right)=0 .
\end{aligned}
$$

Integrating this relation over the interval $\left(r_{h}, r\right)$, we obtain the expression

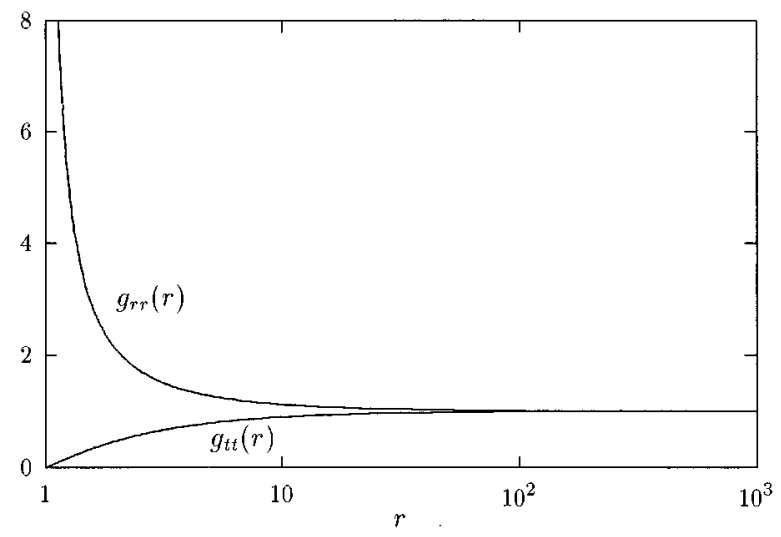

FIG. 2. Metric components $g_{t t}$ and $g_{r r}$ for $r_{h}=1$ black hole.

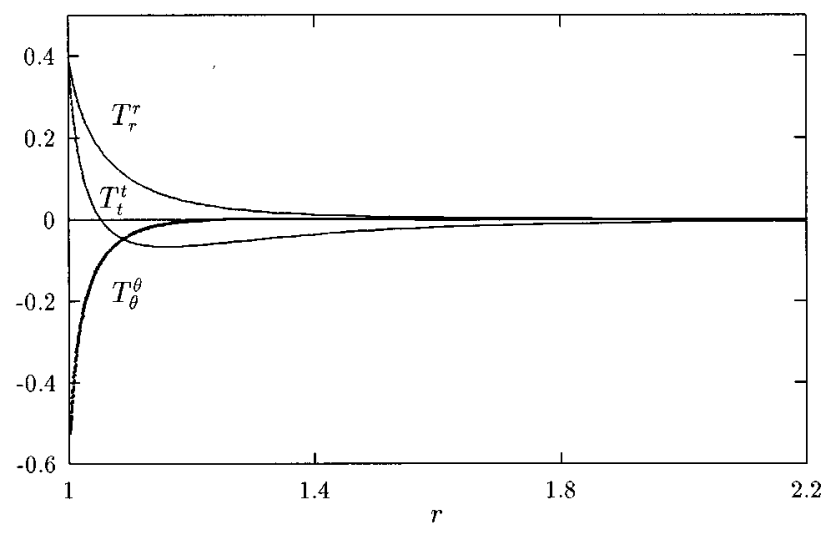

FIG. 3. Components of the energy-momentum tensor for $r_{h}=1$ black hole.

$$
2 M-D=\sqrt{\gamma_{1} \lambda_{1}}\left(r_{h}^{2}+\frac{\alpha^{\prime} e^{\phi_{h}}}{g^{2}}\right) \text {. }
$$

This equation is simply a connection between the set of parameters describing the solution near the horizon and the set $M$ and $D$. The RHS of this relation clearly indicates that the existing dependence of the dilaton charge on the mass does not take the simple form of an equality encountered in Einstein-Yang-Mills-dilaton (EYMD) regular solutions of Ref. [9]. In order to find the relation between $M$ and $D$ we follow Refs. [6,7] and take into account the $O\left(\alpha^{\prime 2}\right)$ expression of the dilaton charge in the limit $r \rightarrow \infty$ :

$$
\begin{aligned}
\phi(r) & =\phi_{\infty}+\frac{D}{r}+\cdots \\
& =\phi_{\infty}+\left(\frac{e^{\phi_{\infty}}}{2 M} \frac{\alpha^{\prime}}{g^{2}}+\frac{73 e^{2 \phi_{\infty}}}{60(2 M)^{3}} \frac{\alpha^{\prime}}{g^{4}}\right) \frac{1}{r}+\cdots
\end{aligned}
$$

This relation can be checked numerically. The result is shown in Fig. 4. Any deviations from this relation are due to higher-order terms which turn out to be small. The above relation (41) implies that the dilaton hair of the black hole solution, discussed in this section, is a kind of "secondary hair,' in the terminology of Ref. [11]. This hair is generated

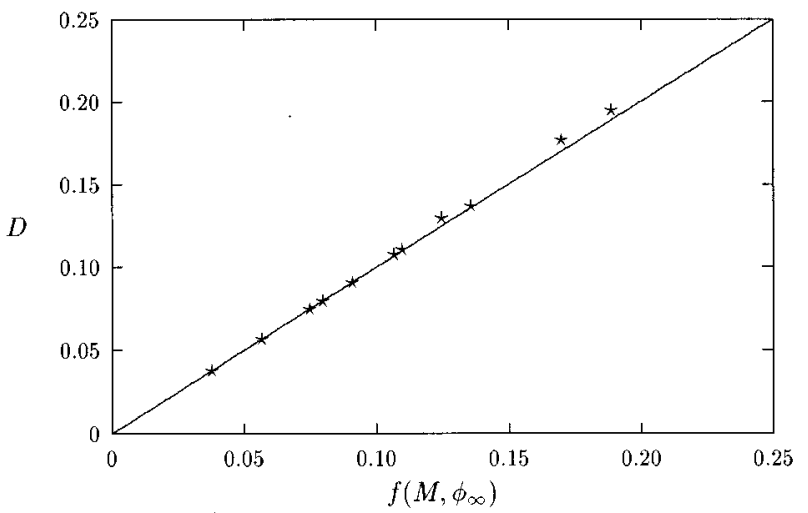

FIG. 4. Dependence of the dilaton charge $D$ on $M$ and $\phi_{\infty}$ for the $r_{h}=1$ black hole. The function $f\left(M, \phi_{\infty}\right)$ stands for the coefficient of $1 / r$ in Eq. (41). 


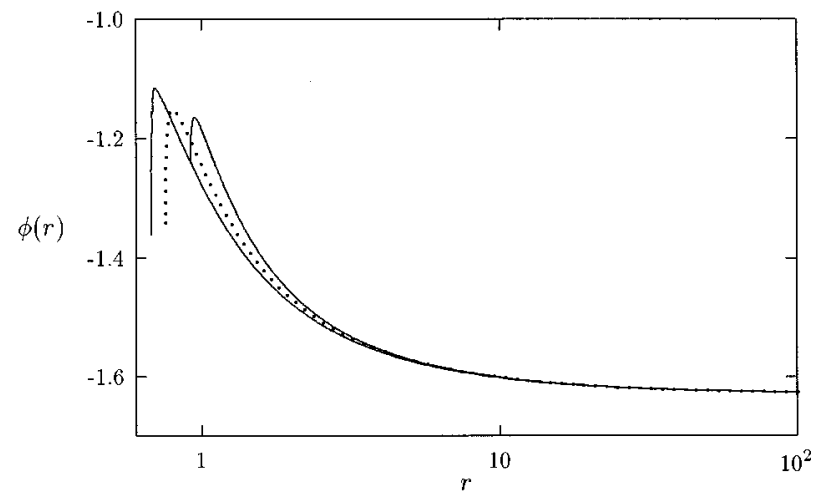

FIG. 5. Dilaton field for the singular solution (42). Each curve corresponds to a different solution characterized by a different value of $r_{s}\left(r_{s}=0.92,0.75,0.62\right)$.

because the basic fields (gravitons) of the theory associated with the primary hair (mass) act as sources for the nontrivial dilaton configurations outside the horizon of the black hole.

\section{ADDITIONAL SOLUTIONS}

A second class of solutions can be obtained if we allow $\phi^{\prime}(r)$ to be infinite at some finite value $r_{s}$ of the coordinate $r$. This choice, as we shall argue below, is not incompatible with the finiteness of the energy-momentum tensor. This is due to the fact that the Gauss-Bonnet term does not have a definite signature. These solutions have the same asymptotic characterization in terms of $\phi_{\infty}, M$, and $D$ as the black hole. Near $r \simeq r_{s}$, one obtains

$$
\begin{gathered}
e^{-\Lambda(r)}=\lambda_{1}\left(r-r_{s}\right)+\cdots, \\
\Gamma^{\prime}(r)=\frac{\gamma_{1}}{\sqrt{r-r_{s}}}+\cdots, \\
\phi(r)=\phi_{s}+\phi_{s}^{\prime} \sqrt{r-r_{s}}+\cdots .
\end{gathered}
$$

To lowest order, the equations of motion yield the constraints

$$
\frac{\alpha^{\prime}}{4 g^{2}} e^{\phi_{s}} \phi_{s}^{\prime} \gamma_{1}=\frac{1}{\lambda_{1}}+\frac{\phi_{s}^{\prime 2} r_{s}^{2}}{16}
$$

and

$$
a \gamma_{1}^{2}+b \gamma_{1}+c=0
$$

with

$$
\begin{gathered}
a=16 e^{2 \phi_{s}}\left(2 e^{\phi_{s}} \phi_{s}^{\prime 2}+8 r_{s}+\phi_{s}^{\prime 2} r_{s}^{2}\right) \\
b=4 e^{\phi_{s}}\left(e^{2 \phi_{s}} \phi_{s}^{\prime 3}-4 e^{\phi_{s}} \phi_{s}^{\prime 3} r_{s}^{2}-16 \phi_{s}^{\prime} r_{s}^{3}-2 \phi_{s}^{\prime 3} r_{s}^{4}\right) \\
c=-8 e^{2 \phi_{s}} \phi_{s}^{\prime 2} r_{s}-e^{2 \phi_{s}} \phi_{s}^{\prime 4} r_{s}^{2}+2 e^{\phi_{s}} \phi_{s}^{\prime 4} r_{s}^{4}+8 \phi_{s}^{\prime 2} r_{s}^{5}+\phi_{s}^{\prime 6}
\end{gathered}
$$

These apparently singular solutions comprise a twoparameter family. The behavior of the dilaton field and the metric components is shown in Figs. 5 and 6, respectively.

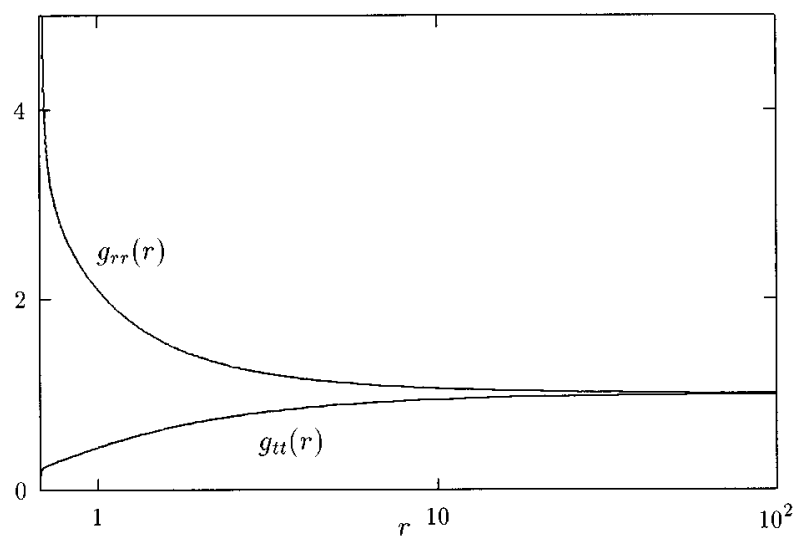
(42)

FIG. 6. Metric components for the $r_{s}=0.68$ singular solution

As we can see, these solutions cannot be classified as black hole solutions since the metric component $g_{t t}$ does not exhibit any singular behavior: $e^{\Gamma} \rightarrow$ const when $r \rightarrow r_{s}$. Only $g_{r r}$ takes on an infinite value when $r_{s}$ is approached.

In order to determine whether the space-time geometry is really singular at $r_{s}$, the scalar curvature $R$ as well as the "curvature invariant" $I=R^{\mu \nu \rho \sigma} R_{\mu \nu \rho \sigma}$ were calculated. It turns out that none of the above quantities exhibits any singular behavior at $r_{s}$ which implies that the pathology of the metric is due to a pathology of the coordinate system and not due to the space-time geometry itself. Moreover, this guarantees the finiteness of the action (it can be easily checked that the Gauss-Bonnet combination is also finite which is consistent with the field redefinition ambiguity arguments given in the introduction). It is a simple exercise to verify that the components of the energy-momentum tensor are also finite. They are shown in Fig. 7. Unfortunately, at present, we are not in a position to discuss the nature of the solutions for $r \leqslant r_{s}$, and hence the only safe conclusion to be made from the above analysis concerns the absence of a naked singularity.

It is interesting to mention the existence of another class of solutions, which are regular in the metric, do not possess any horizon, but the dilaton becomes infinite at $r \simeq 0$. Near the origin these solutions are

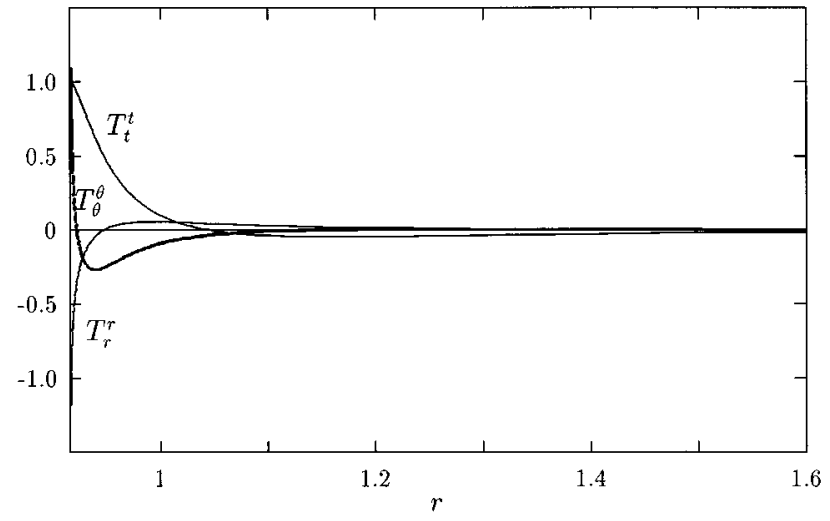

FIG. 7. Components of the energy-momentum tensor for the $r_{s}=0.92$ singular solution (42). 


$$
\begin{gathered}
e^{\Lambda(r)}=1+\lambda_{1} e^{-4 \gamma_{1} / r}, \\
\Gamma^{\prime}(r)=\gamma_{1} e^{-\phi_{1} / r}, \\
\phi(r)=\frac{\phi_{1}}{r}+\cdots .
\end{gathered}
$$

This is also a two-parameter family of solutions. The far asymptotic behavior is still given by Eqs. (20)-(22), and it is again characterized by the parameters $\phi_{\infty}, M$, and $D$. These solutions appear to have no curvature singularities $[R(r \simeq 0) \simeq 0]$, but the components of the energy-momentum tensor are infinite at $r \simeq 0$.

Note that our black hole solution appears to be a boundary surface in the phase space between solutions (42) and (46). This means that, if $\phi_{0}, \phi_{0}^{\prime}, \Gamma_{0}$, and $\Gamma_{0}^{\prime}$ are the values of the fields for the black hole solution at $r=r_{0} \gg 1$, then integration of the system (A3) and (A4), starting from $r_{0}$ with $\phi^{\prime}\left(r_{0}\right)>\phi_{0}^{\prime}$, leads to the solution (42), while the case $\phi^{\prime}\left(r_{0}\right)<\phi_{0}^{\prime}$ leads to the solution (46).

\section{CONCLUSIONS AND OUTLOOK}

In this paper we have dealt with solutions of the coupled dilaton-graviton system in four dimensions, in the presence of higher-curvature terms in the Gauss-Bonnet combination. We have demonstrated the existence of black hole solutions for this system, characterized by nontrivial scalar (dilaton) hair. This hair is of "secondary"-type, in the sense that it is not accompanied by the presence of any new quantity that characterizes the black hole. Indeed, it was shown above that the dilaton charge is not an independent quantity, but it can be expressed in terms of the mass of the black hole. It should be stressed, however, that irrespective of the precise type of hair, the set of solutions examined in this work bypasses the conditions of the no-hair theorem [4]. Thus, our solutions may be viewed as demonstrating that there is plenty of room in the gravitational structure of superstring theory to allow for physically sensible situations that are not covered by the theorem as stated. Although our results were derived in the framework of the $O\left(\alpha^{\prime}\right)$ effective superstring action, they are nonperturbative in nature and they will persist at least in situations of moderate curvatures.

In addition to the black hole solutions, we were able to find two other families of solutions, one of which had the interesting feature of having finite energy density. At present, the physical significance of the solutions is not fully clear to us. We hope to be able to study these structures in the near future.

There are many features of the solutions which we did not address in this work, one of which is their stability under either linear time-dependent perturbations of the gravitondilaton multiplet, or under generic perturbations (beyond linearity). Such an analysis has been performed for the Einstein-Yang-Mills-Higgs system [5], and one could think of extending it to incorporate higher-curvature gravity theories. A stability analysis, when completed, will prove essential in understanding better the physical significance of the black hole solutions found in this work. This is of particular interest due to the connection of the solutions with superstring theory. We hope to return to these issues in a future publication.

\section{ACKNOWLEDGMENTS}

P.K., N.E.M., and J.R. would like to thank the Theory Division at CERN for the hospitality during the final stages of this work. Two of us (K.T. and P.K.) acknowledge traveling support by the EEC Human Capital and Mobility Network "Flavourdynamics"' (CHRX-CT93-0132). In addition, P.K. acknowledges financial support for traveling to CERN by the Greek Ministry of Technology. N.E.M. thanks P.P.A.R.C. for financial support (United Kingdom). E.W. wishes to thank EPSRC (United Kingdom) for financial support.

\section{APPENDIX}

We shall consider now our system of equations and we shall investigate the existence of solutions under the general assumptions $\Gamma^{\prime} \rightarrow \infty$ and $\phi, \phi^{\prime}$ finite when $r \rightarrow r_{h}$. We are not going to make use of any ansatz as the one employed in the previous section since we intend to derive the most general existing solution under the above assumptions. For calculational convenience we set $\alpha^{\prime} / g^{2} \rightarrow 1$, shifting $\phi \rightarrow \phi-\ln \left(\alpha^{\prime} / g^{2}\right)$. We start by observing that the $(r r)$ component can be solved analytically to yield an expression for $e^{\Lambda}$ :

$$
e^{\Lambda}=\frac{-\beta+\delta \sqrt{\beta^{2}-4 \gamma}}{2}, \quad \delta= \pm 1
$$

where

$$
\begin{gathered}
\beta=\frac{\phi^{\prime 2} r^{2}}{4}-1-\Gamma^{\prime}\left(r+\frac{e^{\phi} \phi^{\prime}}{2}\right), \\
\gamma=\frac{3}{2} \Gamma^{\prime} \phi^{\prime} e^{\phi} .
\end{gathered}
$$

We then eliminate $\Lambda^{\prime}$ using $(d / d r)(r r)$. Choosing two of the remaining Eqs. (10), (11), and (13) (only two of them are linearly independent), we obtain the system of equations

$$
\begin{gathered}
\phi^{\prime \prime}=-\frac{d_{1}}{d}, \\
\Gamma^{\prime \prime}=-\frac{d_{2}}{d},
\end{gathered}
$$

where $d, d_{1}$, and $d_{2}$ are given by 


$$
\begin{aligned}
& d=4 e^{2 \Lambda+\phi} r\left(-4+8 e^{\Lambda}-4 e^{2 \Lambda}-4 \Gamma^{\prime} r+4 \Gamma^{\prime} e^{\Lambda} r+5 \phi^{\prime 2} r^{2}-\phi^{\prime 2} e^{\Lambda} r^{2}\right)+4 \phi^{\prime} e^{\Lambda+2 \phi}\left(6-12 e^{\Lambda}+6 e^{2 \Lambda}+6 \Gamma^{\prime} r-8 \Gamma^{\prime} e^{\Lambda} r\right. \\
& \left.+2 \Gamma^{\prime} e^{2 \Lambda} r-3 \phi^{\prime 2} r^{2}+\phi^{\prime 2} e^{\Lambda} r^{2}\right)-12 \Gamma^{\prime} \phi^{\prime 2} e^{3 \phi}\left(1-e^{\Lambda}\right)^{2}-8 \phi^{\prime} e^{3 \Lambda} r^{4} \\
& d_{1}=2 \Gamma^{\prime} \phi^{\prime 3} e^{3 \phi}\left(9 \Gamma^{\prime}-6 \phi^{\prime}-6 \Gamma^{\prime} e^{\Lambda}+12 \phi^{\prime} e^{\Lambda}+\Gamma^{\prime} e^{2 \Lambda}-6 \phi^{\prime} e^{2 \Lambda}\right)+\phi^{\prime 2} e^{\Lambda+2 \phi}\left(24 \phi^{\prime}-8 \Gamma^{\prime} e^{\Lambda}-48 \phi^{\prime} e^{\Lambda}+8 \Gamma^{\prime} e^{2 \Lambda}\right. \\
& +24 \phi^{\prime} e^{2 \Lambda}-42 \Gamma^{\prime 2} r-30 \phi^{\prime 2} r+20 \Gamma^{\prime 2} e^{\Lambda} r-32 \Gamma^{\prime} \phi^{\prime} e^{\Lambda} r+16 \phi^{\prime 2} e^{\Lambda} r-2 \Gamma^{\prime 2} e^{2 \Lambda} r-2 \phi^{\prime 2} e^{2 \Lambda} r+3 \Gamma^{\prime} \phi^{\prime 2} r^{2} \\
& \left.-3 \Gamma^{\prime} \phi^{\prime 2} e^{\Lambda} r^{2}+24 \Gamma^{\prime} \phi^{\prime} r+8 \Gamma^{\prime} \phi^{\prime} e^{2 \Lambda} r\right)+\phi^{\prime} e^{2 \Lambda+\phi}\left(-24+48 e^{\Lambda}-24 e^{2 \Lambda}-4 \Gamma^{\prime} r-16 \phi^{\prime} r+8 \Gamma^{\prime} e^{\Lambda} r+32 \phi^{\prime} e^{\Lambda} r\right. \\
& -4 \Gamma^{\prime} e^{2 \Lambda} r-16 \phi^{\prime} e^{2 \Lambda} r+32 \Gamma^{\prime 2} r^{2}-16 \Gamma^{\prime} \phi^{\prime} r^{2}+38 \phi^{\prime 2} r^{2}+16 \Gamma^{\prime} \phi^{\prime} e^{\Lambda} r^{2}-6 \phi^{\prime 2} e^{\Lambda} r^{2}-3 \Gamma^{\prime} \phi^{\prime 2} r^{3}+\Gamma^{\prime} \phi^{\prime 2} e^{\Lambda} r^{3} \\
& \left.-8 \Gamma^{\prime 2} e^{\Lambda} r^{2}\right)+2 e^{3 \Lambda} r\left(8-16 e^{\Lambda}+8 e^{2 \Lambda}+4 \Gamma^{\prime} r-4 \Gamma^{\prime} e^{\Lambda} r-4 \Gamma^{\prime 2} r^{2}-6 \phi^{\prime 2} r^{2}+\Gamma^{\prime} \phi^{\prime 2} r^{3}-2 \phi^{\prime 2} e^{\Lambda} r^{2}\right) \text {, } \\
& d_{2}=\Gamma^{\prime} \phi^{\prime} e^{\Lambda+2 \phi} r\left(18 \Gamma^{\prime 2}+6 \phi^{\prime 2}-4 \Gamma^{\prime 2} e^{\Lambda}+8 \phi^{\prime 2} e^{\Lambda}+2 \Gamma^{\prime 2} e^{2 \Lambda}+2 \phi^{\prime 2} e^{2 \Lambda}+5 \Gamma^{\prime} \phi^{\prime 2} e^{\Lambda} r-8 \phi^{\prime 3} e^{\Lambda} r-9 \Gamma^{\prime} \phi^{\prime 2} r\right) \\
& -2 \Gamma^{\prime 3} \phi^{\prime 2} e^{3 \phi}\left(3+e^{2 \Lambda}\right)+\phi^{\prime} e^{3 \Lambda} r^{2}\left(8-8 e^{\Lambda}-4 \Gamma^{\prime} r-4 \Gamma^{\prime} e^{\Lambda} r-4 \Gamma^{\prime 2} r^{2}-2 \phi^{\prime 2} r^{2}+\Gamma^{\prime} \phi^{\prime 2} r^{3}\right)+e^{2 \Lambda+\phi}\left(8 \Gamma^{\prime}-16 \Gamma^{\prime} e^{\Lambda}\right. \\
& +8 \Gamma^{\prime} e^{2 \Lambda}-4 \Gamma^{\prime 2} r+8 \phi^{\prime 2} r+8 \Gamma^{\prime 2} e^{\Lambda} r-4 \Gamma^{\prime 2} e^{2 \Lambda} r-8 \phi^{\prime 2} e^{2 \Lambda} r-12 \Gamma^{\prime 3} r^{2}-10 \Gamma^{\prime} \phi^{\prime 2} r^{2}-8 \phi^{\prime 3} r^{2}+4 \Gamma^{\prime 3} e^{\Lambda} r^{2} \\
& +2 \Gamma^{\prime} \phi^{\prime 2} e^{\Lambda} r^{2}+8 \phi^{\prime 3} e^{\Lambda} r^{2}+13 \Gamma^{\prime 2} \phi^{\prime 2} r^{3}+4 \Gamma^{\prime} \phi^{\prime 3} r^{3}+6 \phi^{\prime 4} r^{3}+4 \Gamma^{\prime} \phi^{\prime 3} e^{\Lambda} r^{3}-2 \phi^{\prime 4} e^{\Lambda} r^{3}-3 \Gamma^{\prime} \phi^{\prime 4} r^{4} \\
& \left.-3 \Gamma^{\prime 2} \phi^{\prime 2} e^{\Lambda} r^{3}\right) \text {. }
\end{aligned}
$$

Assuming $\phi_{h}$ and $\phi_{h}$ to be finite and $\Gamma^{\prime} \rightarrow \infty$ when $r \rightarrow r_{h}$, we expand the RHS of Eq. (A1) near the horizon

$$
\begin{aligned}
e^{\Lambda}= & \frac{1}{2}\left(e^{\phi} \phi^{\prime}+2 r\right) \Gamma^{\prime}-\frac{8 e^{\phi} \phi^{\prime}-8 r+e^{\phi} \phi^{\prime 3} r^{2}+2 \phi^{\prime 2} r^{3}}{4\left(e^{\phi} \phi^{\prime}+2 r\right)} \\
& +O\left(\frac{1}{\Gamma^{\prime}}\right)
\end{aligned}
$$

for $\left(e^{\phi} \phi^{\prime}+2 r\right) \neq 0$ and $\delta=1 .^{2}$ Substituting Eq. (A5) in Eqs. (A3) and (A4), we obtain

$$
\begin{gathered}
\phi^{\prime \prime}=-\frac{1}{2} \frac{\left(e^{\phi} \phi^{\prime}+2 r\right)\left(6 e^{\phi}+e^{\phi} \phi^{\prime 2} r^{2}+2 \phi^{\prime} r^{3}\right)}{-6 e^{2 \phi}+e^{\phi} \phi^{\prime} r^{3}+2 r^{4}} \Gamma^{\prime}+1, \\
\Gamma^{\prime \prime}=-\frac{-6 e^{2 \phi}+e^{2 \phi} \phi^{\prime} r^{2}+4 e^{\phi} \phi^{\prime} r^{3}+4 r^{4}}{-6 e^{2 \phi}+e^{\phi} \phi^{\prime} r^{3}+2 r^{4}} \Gamma^{\prime 2}+O\left(\Gamma^{\prime}\right) .
\end{gathered}
$$

We now observe that in order to keep $\phi_{h}^{\prime \prime}$ finite we have to impose the boundary condition $6 e^{\phi}+e^{\phi} \phi^{\prime 2} r^{2}+2 \phi^{\prime} r^{3}=0$ which relates $\phi_{h}^{\prime}$ with $\phi_{h}$ :

\footnotetext{
${ }^{2}$ This has to be understood by the following facts: (i) the choice $\delta=-1$ leads to $e^{\Lambda}=1$ near the horizon, which is not a black hole solution, and (ii) if $\left(e^{\phi} \phi^{\prime}+2 r\right) \simeq 0$, then one obtains $e^{\Lambda}$ $=\sqrt{3 r}\left(\Gamma^{\prime}\right)^{1 / 2}+\cdots$ and $\phi^{\prime \prime}=\sqrt{3 r} e^{-\phi}\left(\Gamma^{\prime}\right)^{1 / 2}+\cdots$ which implies that $\phi_{h}^{\prime \prime}$ is finite at the horizon only if $\phi_{h} \rightarrow \infty$. This is inconsistent with our initial assumption for finite $\phi_{h}$ and $\phi_{h}^{\prime}$.
}

$$
\begin{aligned}
& \phi_{h}^{\prime}=r_{h} e^{-\phi_{h}}\left(-1+\sigma \sqrt{1-6 \frac{e^{2 \phi_{h}}}{r_{h}^{4}}}\right), \quad \sigma= \pm 1, \\
& \phi_{h}<\ln \left(\frac{r_{h}^{2}}{\sqrt{6}}\right)
\end{aligned}
$$

and implies

$$
\begin{gathered}
\phi^{\prime \prime}=1 \\
\Gamma^{\prime \prime}=-\Gamma^{\prime 2}+1 \Rightarrow \Gamma^{\prime}=\frac{1}{r-r_{h}}+1
\end{gathered}
$$

Note that this "black-hole"-type solution is unique and has resulted directly from the requirement $\phi, \phi^{\prime}, \phi^{\prime \prime}$ finite for $\Gamma^{\prime} \rightarrow \infty$. Equivalently,

$$
\begin{gathered}
e^{\Gamma(r)}=\gamma_{1}\left(r-r_{h}\right)+O\left(r-r_{h}\right)^{2}, \\
e^{-\Lambda(r)}=\lambda_{1}\left(r-r_{h}\right)+O\left(r-r_{h}\right)^{2},
\end{gathered}
$$

which is exactly the ansatz (25) used in Sec. II. $\gamma_{1}$ is an arbitrary constant, and $\lambda_{1}=2 /\left(e^{\phi_{h}} \phi_{h}^{\prime}+2 r_{h}\right)$. Notice that Eq. (A8) is exactly the Eq. (35), obtained previously, from the dilaton equation of motion near the horizon. This shows that if Eq. (A8) is satisfied, then not only the finiteness of $\phi_{h}^{\prime \prime}$ is guaranteed but also the expected singular behavior of the metric is assured. The above analysis leads to the conclusion that the asymptotic solution (A8)-(A10), is the only acceptable black hole solution with finite $\phi_{h}, \phi_{h}^{\prime}$ and $\phi_{h}^{\prime \prime}$.
[1] For a review see M. Green, J. Schwarz, and E. Witten, Superstring Theory (Cambridge University Press, Cambridge, England, 1987).

[2] A. Shapere, S. Trivedi, and F. Wilczek, Mod. Phys. Lett. A 6,
2677 (1991); K. Lee and E. J. Weinberg, Phys. Rev. D 44, 3159 (1991); S. G. Ridgway and E. J. Weinberg, ibid. 52, 3440 (1995); A. Garcia, D. Galtsov, and O. Kechkin, Phys. Rev. Lett. 74, 1276 (1995); A. Achucarro, R. Gregory, and K. 
Kuijken, Phys. Rev. D 52, 5729 (1995); S. G. Turyshev, Report No. hep-th/9509101 (unpublished); T. Matos, D. Nunez, and H. Quevedo, Phys. Rev. D 51, 310 (1995).

[3] P. Bizon, Phys. Rev. Lett. 64, 2844 (1990); K. Lee, V. P. Nair, and E. J. Weinberg, Phys. Rev. D 45, 2751 (1992); B. R. Greene, S. D. Mathur, and C. M. O’Neil, ibid. 47, 2242 (1993); G. E. Lavrelashvili and D. Maison, Phys. Lett. B 295, 67 (1992); Nucl. Phys. B410, 407 (1993); P. Breitenlohner, P. Forgacs, and D. Maison, ibid. B383, 367 (1992); Commun. Math. Phys. 163, 141 (1994); Nucl. Phys. B442, 126 (1995); P. C. Aichelburg and P. Bizon, Phys. Rev. D 48, 607 (1993); E. E. Donets and D. V. Gal'tsov, Phys. Lett. B 302, 411 (1993); 312, 392 (1993); K. Torii and K. Maeda, Phys. Rev. D 48, 1643 (1993); C. M. O’Neil, ibid. 50, 865 (1994); B. Kleihaus, J. Kunz, and A. Sood, Phys. Lett. B 372, 204 (1996).

[4] J. D. Bekenstein, Phys. Rev. D 5, 1239 (1972); 5, 2403 (1972); 51, R6608 (1995), and references therein.
[5] N. E. Mavromatos and E. Winstanley, Phys. Lett. B 352, 242 (1995); Phys. Rev. D 53, 3190 (1996).

[6] C. G. Callan, R. C. Myers, and M. J. Perry, Nucl. Phys. B311, 673 (1989); B. Campbell, M. Duncan, N. Kaloper, and K. A. Olive, Phys. Lett. B 251, 34 (1990); B. Campbell, N. Kaloper, and K. A. Olive, ibid. 263, 364 (1991); 285, 199 (1992); P. Kanti and K. Tamvakis, Phys. Rev. D 52, 3506 (1995).

[7] S. Mignemi and N. R. Stewart, Phys. Rev. D 47, 5259 (1993); S. Mignemi, ibid. 51, 934 (1995).

[8] R. Metsaev and A. Tseytlin, Nucl. Phys. B293, 385 (1987).

[9] E. Donets and D. Gal'tsov, Phys. Lett. B 352, 261 (1995).

[10] G. Gibbons and K. Maeda, Nucl. Phys. B298, 741 (1988); D. Garfinkle, G. Horowitz, and A. Strominger, Phys. Rev. D 43, 3140 (1991); 45, 3888 (1992); A. Gosh and P. Mitra, Phys. Rev. Lett. 73, 2521 (1994).

[11] S. Coleman, J. Preskill, and F. Wilczek, Nucl. Phys. B378, 175 (1992). 